\title{
Meta
}

Journal des traducteurs

Translators' Journal

\section{GLADSTONE, W.J. (1990) : Dictionnaire anglais-français des sciences médicales et paramédicales, 3 éd., Saint-Hyacinthe, Edisem, Paris, Maloine, 1099 p.}

\section{Louise Le Blanc}

Volume 36, numéro 4, décembre 1991

URI : https://id.erudit.org/iderudit/003431ar

DOI : https://doi.org/10.7202/003431ar

Aller au sommaire du numéro

Éditeur(s)

Les Presses de l'Université de Montréal

ISSN

0026-0452 (imprimé)

1492-1421 (numérique)

Découvrir la revue

Citer ce compte rendu

Le Blanc, L. (1991). Compte rendu de [GLADSTONE, W.J. (1990) : Dictionnaire

anglais-français des sciences médicales et paramédicales, $3^{\mathrm{e}}$ éd.,

Saint-Hyacinthe, Edisem, Paris, Maloine, 1099 p.] Meta, 36(4), 665-667.

https://doi.org/10.7202/003431ar d'utilisation que vous pouvez consulter en ligne.

https://apropos.erudit.org/fr/usagers/politique-dutilisation/ 
GLADSTONE, W.J. (1990): Dictionnaire anglais-français des sciences médicales et paramédicales, $3^{\mathrm{e}}$ éd., Saint-Hyacinthe, Edisem, Paris, Maloine, 1099 p.

Il s'agit de la troisième édition du Dictionnaire anglais-français des sciences médicales et paramédicales de W. J. Gladstone. La première avait paru en 1978 et la seconde en 1984. Cette $3^{\mathrm{e}}$ édition présente les mêmes caractéristiques que les deux premières. Précédées des préfaces des $1^{\mathrm{re}}$ et $3^{\mathrm{e}}$ éditions, (la préface de la présente édition a été rédigée par Madame Amal Jammal, de l'Université de Montréal), des avant-propos aux $2^{\mathrm{e}}$ et $3^{\mathrm{e}}$ éditions, d'une introduction ainsi que d'une liste des abréviations utilisées dans le texte, les entrées, classées par ordre alphabétique, sont suivies d'une liste d'abréviations, de sigles et de symboles couramment employés en médecine ou dans les domaines connexes. Chaque terme figurant au dictionnaire est accompagné d'une 
abréviation indiquant la catégorie grammaticale à laquelle il appartient et, parfois, de marques d'usage relatives à la région du globe, au niveau de langue ou au domaine où il est employé. En outre, dans certains cas, un contexte illustre son utilisation.

Dans l'avant-propos à cette 3e édition, on affirme que l'ouvrage comporte 3800 additions, modifications ou corrections par rapport à l'édition précédente, sans toutefois préciser le nombre de mots nouveaux qui sont venus enrichir le dictionnaire, ni la nature des modifications et des corrections qui ont été faites. Par conséquent, il est difficile de déterminer jusqu'à quel point cette nouvelle édition diffère des précédentes. Cependant, l'une des améliorations apportées est évidente: la correction des erreurs touchant l'ordre alphabétique, relativement nombreuses dans la $2^{\mathrm{e}}$ édition, facilite grandement la consultation de l'ouvrage. Signalons aussi que l'utilisation de caractères d'imprimerie légèrement plus petits a permis de réduire le format de l'ouvrage sans rendre la lecture plus difficile pour autant.

On peut quand même avoir une idée, bien relative il est vrai, des termes nouveaux qui se sont ajoutés à ce dictionnaire en comparant certaines entrées de la $3^{\mathrm{e}}$ édition aux mêmes entrées de l'édition précédente. On relève ainsi 40 nouveaux termes à l'entrée disease (anterior horn cell disease, calcium pyrophosphate deposition disease, inclusion cell disease, macrovascular disease, subcutaneous spherulocystic disease, etc.), 24 à l'entrée test(n) (cis-trans test, microlymphocyte cytotoxicity test, osmotic fragility test, tail-flick test, etc.), 11 à l'entrée cell (epithelial cell of convoluted tubule, prickle cell, wild-type cell, etc.), 10 à l'entrée system (dense tubular system, marginal microtubular system, orthorhombic system, etc.), 5 à l'entrée assay (automated fluorochromatic (or fluorochrome) cytotoxicity assay, colony forming assay, etc.), 5 à l'entrée reaction (catastrophic reaction, $\mathbf{P F}-3$ release reaction, etc.), 3 à l'entrée scale (Glasgow coma scale, sliding scale, time scale) et 2 à l'entrée scanning (fan-shaped scanning, ventilation-perfusion scanning). On constate également qu'un grand nombre de nouveaux syntagmes appartenant aux domaines de la génétique, de la biologie moléculaire et de l'immunologie figurent maintenant au Gladstone, ce qui reflète bien l'essor qu'ont connu ces disciplines au cours des dernières années. Par exemple, les entrées mutation, gene, genetics, site, probe, antibody se sont enrichies respectivement de 15 (conditional lethal mutation, forward mutation, insertion mutation, etc.) de six (constitutive gene, housekeeping gene, luxury gene, etc.), de trois (behavior genetics, medical genetics, molecular genetics) de quatre (acceptor splicing site, donor splicing site, replacement site, restriction site) de deux (DNA probe, RNA probe) de trois (Donath and Landsteiner antibody, islet-cell antibody, warm antibody) nouveaux syntagmes. On note aussi que les termes suivants, absents des éditions précédentes, se retrouvent maintenant dans la $3^{\text {e }}$ édition de ce dictionnaire: enzyme immunoassay, fluorescence polarization immunoassay, immunoblotting, immunoglobulin disorder, solid phase immunosorbent technique, complementation assay, priming of DNA synthesis, genetic distance, genetic footprint, DNA gap, micronucleus test, DNA repair test, concatenated circles of DNA, writhing number.

Comme les précédentes, cette édition comporte des termes figurant dans la documentation médicale, mais empruntés à d'autres domaines, notamment aux statistiques, à la chimie, au droit, à l'informatique, au commerce etc. Ces renseignements peuvent se révéler particulièrement utiles à la compréhension ainsi qu'à la traduction de nombreux textes, notamment des rapports de recherche dans lesquels on retrouve très souvent des termes de chimie ou de statistique. Voici quelques exemples de termes appartenant aux statistiques, à la chimie et à l'informatique qui viennent d'être inclus dans la présente édition du Gladstone: maximum likelihood test, one-sided test, twotail test, Student's paired t test, affinity chromatography, preparative liquid elution 
chromatography, reverse-phase partition chromatography, anorthic system, monoclinic system, computer graphics.

Dans l'introduction, l'auteur affirme que son ouvrage n'est ni complet ni parfait. Nul doute que certaines erreurs sont inévitables et s'expliquent par l'ampleur de la tâche que constitue la rédaction d'un dictionnaire. La synonymie, entre autres, est parfois discutable. Par exemple, à l'entrée tablet, on retrouve comme équivalents de film-coated tablet: comprimé enrobé, comprimé pelliculé. Or, ces deux termes ne sont probablement pas synonymes, si on en juge par les définitions que donnent certains auteurs. En effet, selon ceux-ci, un comprimé est dit pelliculé seulement si son enrobage est mince. Comprimé enrobé serait donc un terme générique et comprimé pelliculé, un terme spécifique. Il arrive aussi qu'on donne comme équivalents d'un terme générique des termes spécifiques. C'est le cas notamment de pelvic inflammatory disease (GÉN): salpingite aiguë (SPÉC), pelvipéritonite (SPEC). De plus, certains équivalents manquent de précision alors que d'autres ne sont pas tout à fait justes. Ainsi, on propose immunodiffusion par la technique de Laurell comme équivalent de rocket immunoelectrophoresis, sans indiquer de quelle technique il s'agit. Or, Laurell aurait mis au point deux variantes de l'immunoélectrophorèse ${ }^{3}$. L'un des équivalents de enzyme immunoassay serait immunoenzymologie. Cette dernière n'est-elle pas plutôt une branche de l'immunologie qu'une méthode de dosage? Enfin, certains équivalents sont douteux : mutation «down», mutation «leaky». Cependant, même dans les cas où ils sont imparfaits, les équivalents constituent une piste qui orientera le lecteur ou le traducteur dans sa recherche d'une meilleure réponse à ses questions. Il est en effet plus facile d'avoir pour point de départ une solution imparfaite qu'un vide total.

Douze ans se sont donc écoulés depuis la parution de la 1 re édition de cet ouvrage. Malgré certaines imperfections, celui-ci conserve toute son utilité, notamment en raison de la pertinence des syntagmes y figurant. En effet, ces derniers sont ceux que l'on retrouve couramment dans les textes médicaux et paramédicaux les plus récents. Cette pertinence est probablement attribuable à la méthode de travail de l'auteur et de ses collaborateurs. En effet, ceux-ci ne se contentent pas de compiler les dictionnaires existants, mais dépouillent méthodiquement des centaines de textes, rédigés tant en anglais qu'en français, puis établissent la correspondance entre les termes des deux langues. Ils demeurent ainsi sensibles non seulement à l'évolution des disciplines médicales et paramédicales, mais aussi à celle de la langue. Ils sont donc en mesure, d'une édition à l'autre, d'enrichir l'ouvrage de termes nouveaux et d'y apporter les modifications nécessaires. Bref, le Dictionnaire anglais-français des sciences médicales et paramédicales de W. J. Gladstone demeure incontestablement l'outil indispensable de l'usager de la langue médicale.

LOUISE LE BLANC

BIBLIOGRAPHIE

DESCOUTURES, J.M. (1988): Le Bon Usage des médicaments, Paris, Frison-Roche/Sidem, p. 47.

LE HIR, A. (1983) : Abrégé de pharmacie galénique, Paris, Masson, p. 231.

BACH, J.-F., LESAVRE, P. (1989) : Immunologie, Paris, Flammarion, Médecine-Sciences, p. 97. 